\title{
Transition to Sustainable Energy Generation in Australia: Interplay Between Coal, Gas and Renewables
}

\begin{abstract}
This paper analyzes the ongoing transition to sustainable energy in Australia, moving from traditional large-scale plants to distributed renewable generation by studying the time series of coal and gas consumption as well as onshore wind and solar. Even though most of energy generation, especially in the form of electricity is currently being generated from coal and gas, a quantitative assessment of their evolution is necessary to understand whether, and to which extent, renewables are competing in the marketplace with conventional production means. A well-accepted innovation diffusion model is used to capture and interpret the underlying dynamics of the competitive transition in generation. The results show that renewables are exerting a competitive pressure on coal and collaborate with gas towards the transition. The view that gas should play a key role in transition is confirmed by our findings, because it is found to have a competitive role towards coal, while aiding the uptake of renewables.
\end{abstract}

Keywords: energy transition, renewable energy sources, competition models, innovation diffusion 


\section{Introduction}

In order to face climate change and energy security problems, many countries are currently beginning a process of energy transition from non renewable to renewable energy sources. A good example of energy transition is represented by Germany with the so called Energiewende, a process that expects to reach by 2020 the complete nuclear phase out and 35\% of electricity provision by renewable sources. In fact, the term energy transition typically refers to an electricity one, [1], [2]. Of all the countries that are dealing with this regime transformation, an interesting case is represented by Australia. As observed by Byrnes et al. in [3], Australia has a governmental structure similar to that of Germany, which would facilitate to take it as a model to follow; however, factors such as local culture, geographical size, remoteness and abundance of gas and coal, have concurred to determine a substantial difference in the energy agenda of these two countries. Hua et al. [4] reminded that Australia has a competitive advantage in being endowed with abundant fossil fuel reserves, which allow a relatively cheap generation with high emissions. On the other hand, it also has very rich renewable energy sources, including the highest average solar radiation, [3]. Indeed it is expected that green policies will lead in the near future to an increasing share of renewables in the Australian energy mix, mainly in the form of wind and solar, in order to control $\mathrm{CO}_{2}$ emissions. Nowadays, the development of renewables is supported both at national and state levels. The Australian Renewable Energy Target (RET) is the federal government scheme according to which $20 \%$ of electricity should be generated by renewables by 2020 . Since 2011 the RET has been operating in two parts: the large-scale Renew- 
able Energy Target (LRET), creating a financial incentive for the production of electricity from large scale renewable power plants, and the Small Scale Renewable Energy Scheme (SRES), creating financial incentives for home owners to install small-scale renewable systems. On top of the RET, which provides a unified support, individual states have set their own targets and designed feed-in tariff schemes since 2008. For example, South Australia has decided to cover $50 \%$ of the state electricity needs from renewables by 2025 , and already achieved the result in 2017. Despite the heterogeneity in energy mixes and in feed-in tariff mechanisms, their implementation implied a rapid increase of renewables across all the Australian states in the last decade, as suggested in an updated review on Australian incentive mechanisms by Poruschi et al. [5].

Focusing on electricity, the Australia Energy Update 2018 [6] and the Australia 2018 Review by IEA [7] report that shares of onshore wind and solar are continuing to grow, with wind now one third of renewable generation at the country level. Remarkably, thanks to natural resources and a favourable policy landscape, South Australia has registered the highest solar penetration rate per household in the world, [7].

Still, in 2016-17, renewables contributed $16 \%$ of electricity generation in Australia. According to [6] and [7], the largest source of electricity is coal with a share of $63 \%$ of total generation. Brown and black coal rose in 2014-15, after three years of decline, although the country's reliance on it is much lower than at the beginning of the century, when its share was more than $80 \%$. The second source of electricity is gas with a market share of about $20 \%$. The dominant industry view is that gas is a transition mean allowing 
to reduce emissions from power generation through a gradual substitution of coal, as reported in Molyneaux et al. in [8]. Provided this, meeting the Australian Renewable Energy Target objective requires a strong effort in technological terms but also in an institutional, cultural and social dimension. Effendi and Courvisanos [9] identified as barriers to the transition to renewables a general inertia of the industry, which is comfortable with fossil fuels, and the fact that renewables are characterized by low density, intermittent supply and storage problems, which makes them difficult to integrate in a highly centralized structure. Indeed, the challenge of introducing large-scale renewable generation to the tightly-integrated legacy electricity grid should not be underestimated in terms of technological difficulties, which may require costly solutions, as highlighted by Brear et al. [10] and Jeppensen et al. [11]. On the other hand, Warren et al. [12] and Sovacool [13] suggested that the problem is not only technological, but involves aspects of policy, institutions, culture and knowledge. Cheung and Davies in [14] suggested that there exists an inherent contradiction with respect to Australia's energy policy, because, despite the actions towards renewables, there remains an on-going a political disposition to subsidise the fossil fuel industry. Most importantly, Byrnes et al. [3] observed that a key challenge for renewables is a kind of network effect since institutions and consumers face the choice between two competing networks, renewables and non renewables.

Non renewables may benefit from positive externalities due to an existing and well established network, while renewables are hindered by their relative newness. As noticed by Sovacool in [13], there is a tendency of new systems 
to face the lock-in or path dependency of existing ones. Social acceptance, community involvement and adequate incentive measures are essential elements for making renewables a competitive solution with respect to fossil fuels and ensure their integration into the existing energy system, which represents one of the crucial challenges of the energy transition. As highlighted in Geels et al. [15], the transition to low-carbon systems is a complex and multidimensional process, involving interactions and feedbacks among several actors of the society, namely firms, consumers, governments, institutions and the media. Given this complexity, in Geels et al. [15] it is suggested that the analysis of energy transition should take a multi-level perspective, able to consider not only techno-economic aspects, but also social, political and cultural dimensions of the process. As an example, the authors consider the case of Germany, showing that the electricity transition in that country is the result of various phases and interactions among firms and economic actors, consumers/citizens and political institutions. Transition is seen as a socio-technical process implying the gradual substitution of an "incumbent regime" with the widespread adoption of "niche technologies": the case of Germany, along with other countries such as Denmark, Portugal and UK, well shows the central role played by public policy, especially in the form of feed-in tariffs, in driving this process, [15].

In this perspective, Australia appears an fascinating case study, in order to evaluate the competitive capacity of niche technologies, renewables, with respect to the incumbent regime, coal and gas, still dominating the market. An analysis on the evolution of each of these sources appears necessary in order to understand to which extent the energy transition is underway, i.e. 
whether renewables are able to compete in the marketplace with conventional production means, and what is the role played by gas.

This paper studies the interplay between coal, gas and renewables by using available time-series data and considering multivariate innovation diffusion models. These models allow simultaneously describing pairs of consumption time series of energy sources and provide a quantitative measure of possible competition or cooperation effects between them.

A similar methodology was proposed in Guidolin and Guseo [1] and in Furlan and Mortarino [16]. Guidolin and Guseo in [1] proposed the application of innovation diffusion models for competition to the annual time series of consumption of nuclear and renewables (wind and solar energy) in Germany, in order to test empirically the presence of a substitution effect. The obtained results show that renewables exerted a significant and measurable effect in determining the observed decline of nuclear power consumption. In particular, the diffusion of renewables - wind and solar - has been characterized by a high within word-of-mouth, testifying the widespread belief of Germans towards the energy transition. Furlan and Mortarino in [16] applied a competition model to the diffusion dynamics of traditional and clean energy systems in US, Europe, China and India, to see whether the diffusion level of traditional sources sustains or prevents the spread of renewables and vice versa.

The original contribution of the paper relies in the application of the innovation diffusion framework to the Australian energy system, in order to capture its complex internal dynamics and statistically test the existence of competition-cooperation effects among market players. Our attention is de- 
voted to the electricity system since renewables are being integrated into it. The rest of the paper develops accordingly: Section 2 provides an overview of the Australian electricity system, in order to build a suitable context for subsequent result interpretation. Section 3 presents the model employed in subsequent analyses along with some methodological aspects concerning statistical identification and model selection. Section 5 is dedicated to the analysis of competition between energy sources, and in particular the following cases are illustrated: coal versus gas, coal versus renewables and gas versus renewables. Section 5 summarizes the most important findings of the study along with some conclusions based on these.

\section{The Australian electricity system: an overview}

Australian electricity has been mostly provided by large, centralized fossil fuel powered generators. According to the Australia 2018 Review by IEA [7] coal had increased for several decades and started to decline in recent years as a result of lower electricity demand and growth of other power generation sources. Electricity generation from gas more than doubled since 2006 and recently stalled.

In structural terms, there is no nationwide electricity grid due to the distribution of the population centers over large distances, but the National Electricity Market (NEM) covers about $90 \%$ of electricity demand, by connecting states and territories of eastern and southern Australia, namely Queensland, New South Wales, Australian Capital Territory, Victoria, Tasmania and South Australia. Western Australia and the Northern Territory are not connected to the NEM, while Tasmania joined the NEM in May 2005. 
The power mix is different across the states pertaining to NEM. Coal is the main source in the three largest power markets, Queensland, New South Wales and Victoria, while natural gas has its major share in Western Australia and Northern Territory. Regarding renewables, onshore wind power is relevant in South Australia, Tasmania and Victoria, solar power is mainly concentrated in South Australia, and hydropower is the most important source of electricity in Tasmania. The NEM operates the world's longest interconnected power systems between Port Douglas, Queensland and Port Lincoln, South Australia with an end-to-end distance of more than $5000 \mathrm{Km}$. Unlike the European grids, Australian power grid is loosely connected with limited import/export capabilities between individual states. This centralized electricity supply model is facing the major challenge of climate change and emissions, since it is well known that electricity is a major responsible for greenhouse gas emissions, and renewables will play a key role in meeting future generation requirements, [3].

Despite the growing importance of renewables and the market incentives developed to foster their uptake, namely the Renewable Energy Target and state-level schemes, the Australian energy policy has often appeared complex, fragmented and in favour of conventional sources through subsidies for fossil fuel use and production. For instance, in 2015 Federal policy reverted to a pro-coal economy with cuts to alternate and renewable energy government offices, targets and subsidies. The carbon pricing mechanism introduced in 2012, was revoked in 2014. Unlike European countries, both major parties now have shown political positions against carbon pricing, as reported in Brear et al. in [10] and Jeppesen et al. in [11]. In 2015 the Government also 
reduced the Renewable Energy Target from 41,000 GWh per year to 33,000 GWh. Mitchell and Casben in [17] suggested that this move away from previous support given to renewables may be interpreted as a concern for the competitiveness of fossil fuels: a reduced commitment to renewable sources appeared necessary in order to ensure investments in conventional production facilities, increasingly characterized by ageing and inefficiency problems. From a technology perspective, the cheap, coal-based centralized generation from few number of large-scale coal plants that are relatively close to population centers has ensured easier management of electricity grid in Australia. Thanks to mostly energy-wise self-sufficient states and due to the very large distances involved, there is only very limited interstate power transfers. This structure makes integration of renewable generation difficult to the existing legacy grid without significant new infrastructure investment. For example, relying on neighbouring generation centers as in Europe or exploiting diversity of different types of renewable generation, e.g. interplay between wind and solar, are difficult in the Australian context without substantial transmission investments. As reported in [7], the challenge of a secure and resilient electricity sector, while facing a profound transformation process, has been a central topic for policy makers and experts in the field after the state-wide blackout in South Australia occurred in September 2016. Following this event, an Independent Review into the Future Security of the NEM, the "Finkel Review" [18], was released in June 2017 after eight months of investigation and consultation. The Finkel Review identified 50 recommendations to address Australia's "energy trilemma", that is providing affordable, reliable and low emissions electricity for the country. The most important 
recommendation is the introduction of a Clean Energy Target, that should replace the RET in 2020. Other key recommendations are the role of gas as essential for the transition away from carbon generation, an increased grid-wide planning and regional reliability requirements, and a stronger governance in terms of improved data availability and the establishment of a new Energy Security Board. Of the 50 recommendations, 49 have been accepted by the Australian government, while the introduction of the CET is still being discussed.

This overview of the Australian electricity system highlights that the transition is characterized by complexities, mostly due to controversial policy choices. All these aspects form the context for a meaningful interpretation of the analysis we develop in the following sections.

\section{Methodology: innovation diffusion models}

A well accepted methodology to study market evolution in the energy domain is the innovation diffusion framework. In fact, new energy sources may be considered as new products needing to be accepted in a market on the basis of consumption choices of final users and market mechanisms such as incentive measures and prices. This approach dates back to the 1970s with Marchetti (see for instance [19], [20], [21]), who proposed the idea that the substitution dynamics occurring in energy systems are similar to those involving other commercial products or technologies. In particular, a central role is played by learning, spread of knowledge and imitation among consumers, that are considered as the real drivers of change. Despite similarities, the technical features of renewables imply some important differences. 
In a recent review on the application of diffusion models to renewables proposed by Rao and Kishore [22], it has been noticed that renewables' typical characteristics such as low load factor, need for energy storage, small size, high upfront costs create a competitive disadvantage, needing focused policies to stimulate the diffusion process. Meade and Islam [23] suggested that renewable technologies are different from other industrial technological innovations because, in the absence of focused support, they are not convenient from a financial point of view. However, the economic and financial aspect is just one of the dimensions to consider: as highlighted by Geels et al. in [15], low-carbon transitions are not only about market diffusion of new technologies but also about changes in life styles, practices, culture and political positions, and are very context specific, depending on socio-economic, technological and institutional factors, as observed by Davies and Diaz-Rainey in [24].

The innovation diffusion framework is a valuable tool for performing a historical data-based analysis, capturing the multidimensional nature of the regime shift.

\subsection{A diffusion model for duopolistic competition}

A well known model to analyze market penetration, also in the energy context, is the Bass model, in the standard [25] and generalized [26] versions. For recent reviews on the use of the Bass models in energy contexts see for instance [1] and [22]. The Bass model, BM, describes and forecasts the development over time of a new product growth, as result of the choices of two groups of adopters, the innovators and the imitators. In particular, innovators are influenced by institutional communication, while imitators by 
social interactions and spread of knowledge. The formal representation of the $\mathrm{BM}$ is a first order differential equation

$$
z^{\prime}(t)=\left[p+q \frac{z(t)}{m}\right][m-z(t)]
$$

where the variation over time of adoptions, $z^{\prime}(t)$, is proportional to the residual market, $m-z(t)$, with $m$ the constant market potential and $z(t)$ cumulative adoptions at time $t$. Parameter $p$ represents the effect of the external information, and defines the behavior of innovators, while $q$ is the coefficient of imitation, and describes the behavior of imitators, whose influence is modulated over time by the ratio $z(t) / \mathrm{m}$.

The Bass model is a suitable choice for forecasting energy markets evolution, thanks to its flexibility and interpretability. Moreover, the generalized Bass model, GBM, by allowing the inclusion of a general intervention function $x(t)$, is able to capture the possible effect of external actions, such as incentive measures. These features provide significant advantages over traditional time series models, like ARIMA structures, or 'black-box' methods widely used in data analysis, which have less interpretation power.

One of the drawbacks of the analyses performed with the Bass model, in standard and generalized versions, is the fact of considering only univariate processes, thus not taking into account a likely competitive environment. However, diffusion models for competition are a quite recent achievement and the literature on the topic is still under development, due to the inherent difficulty of dealing with systems of differential equations.

So far, competition modeling has essentially considered duopolistic conditions, where competitors may be simultaneous or enter the market at differ- 
ent times. The second case, called diachronic competition, was treated in literature by [27], [28], [29], [30], [31]. In this paper we employ the general model for a diachronic duopolistic competition proposed by Guseo and Mortarino in [29], called unbalanced competition and regime change diachronic model, UCRCD. In this model the market potential may take different levels: $m_{a}$, the market potential of the first entrant in the stand-alone phase, and $m_{c}$, the global or category potential under competition. The residual market $m-z(t)$ is assumed to be a common target for each competitor, with $(t)=z_{1}(t)+z_{2}(t)$ denoting common cumulative adoptions and $z_{i}(t), i=1,2$ the cumulative sales of technology $i$. The second competitor enters the market at time $t=c_{2}$ with $c_{2}>0$.

The model is a system of differential equations where $z_{1}^{\prime}(t)$ and $z_{2}^{\prime}(t)$ indicate instantaneous adoptions of the first and of the second market player, respectively, and $I_{A}$ is an indicator function of event $A$,

$$
\begin{aligned}
z_{1}^{\prime}(t)= & m\left\{\left[p_{1 a}+q_{1 a} \frac{z(t)}{m}\right]\left(1-I_{t>c_{2}}\right)\right. \\
& \left.+\left[p_{1 c}+\left(q_{1 c}+\delta\right) \frac{z_{1}(t)}{m}+q_{1 c} \frac{z_{2}(t)}{m}\right] I_{t>c_{2}}\right\}\left[1-\frac{z(t)}{m}\right], \\
z_{2}^{\prime}(t)= & m\left[p_{2}+\left(q_{2}-\gamma\right) \frac{z_{1}(t)}{m}+q_{2} \frac{z_{2}(t)}{m}\right]\left[1-\frac{z(t)}{m}\right] I_{t>c_{2}}, \\
m= & m_{a}\left(1-I_{t>c_{2}}\right)+m_{c} I_{t>c_{2}} \\
z(t)= & z_{1}(t)+z_{2}(t) I_{t>c_{2}} .
\end{aligned}
$$

We may observe that, as long as $t \leqslant c_{2}$ and the second competitor has not yet entered the market, $z^{\prime} 1(t)$ is described through a standard Bass model with parameters $p_{1 a}, q_{1 a}$, and $m_{a}$. When $t>c_{2}$, both concurrents exist in the 
market and evolve according to their own trajectories, which are influenced by competition. The first is characterized by new parameters: the innovation coefficient under competition, $p_{1 c}$, and the imitative one, referred to the imitation, which is split into two parts, the within imitation coefficient $q_{1 c}+\delta$, modulating internal growth through the ratio $z_{1} / m$, and the cross imitation one, $q_{1 c}$ which is powered by $z_{2} / m$ and measures the effect, in terms of positive or negative word-of-mouth, of the second on the first. The second concurrent has three corresponding parameters: the innovation coefficient $p_{2}$, the within imitation coefficient $q_{2}$, modulating internal adoptions through the ratio $z_{2} / m$, and the cross imitation coefficient $q_{2}-\gamma$, which measures the effect, either positive or negative, of the first entrant on the second. Competition is essentially measured by the cross imitation coefficients $q_{1 c}$ and $q_{2}-\gamma$, which may be either positive or negative. In Table 1 we display the possible cases depending on the sign of the coefficients. In this most general case, divide parameters $\delta$ and $\gamma$ are assumed to be possibly different, and the model is called unrestricted UCRCD. If the restriction $\delta=\gamma$ applies, the model takes a reduced form, called standard UCRCD, see [29]. The constraint $\delta=\gamma$ assumes a symmetric behavior between the two competitors, so that the divide between within- and cross-imitation is the same in both: this implies a substantial symmetry between the two players, so that what is lost by one is exactly gained by the other.

\subsection{Statistical inference and estimation}

The statistical implementation of the models presented in previous section is based on nonlinear least squares (NLS), (see [32]), under a convenient stacking of the two submodels; in particular, we may consider the structure 
314

of a nonlinear regression model

$$
w(t)=\eta(\beta, t)+\varepsilon(t),
$$

315

where $w(t)$ is the observed response, $\eta(\beta, t)$ is the deterministic component describing instantaneous or cumulative processes, depending on parameter set $\beta$ and time $t$, and $\varepsilon(t)$ is a residual term, not necessarily independent identically distributed (i.i.d.) The performance of an extended model, $m_{2}$, compared with a nested one, $m_{1}$, may be evaluated through a squared multiple partial correlation coefficient $\tilde{R}^{2}$ in the interval $[0 ; 1]$, namely,

$$
\tilde{R}^{2}=\left(R_{m_{2}}^{2}-R_{m_{1}}^{2}\right) /\left(1-R_{m_{1}}^{2}\right)
$$

where $R_{m_{i}}^{2}, i=1,2$ is the standard determination index of model $m_{i}$.

The $\tilde{R}^{2}$ coefficient has a monotone correspondence with the $F$-ratio, i.e.,

$$
F=\left[\tilde{R}^{2}(n-v)\right] /\left[\left(1-\tilde{R}^{2}\right) u\right]
$$

where $n$ is the number of observations, $v$ the number of parameters of the extended model $m_{2}$, and $u$ the incremental number of parameters from $m_{1}$ to $m_{2}$. Under strong conditions on the distributional shape of the error term $\varepsilon(t)$, particularly i.i.d. and normality, the statistic $F$-ratio, for the null hypothesis of equivalence of the two models, is a central Snedecor's $F$ with $u$ degrees of freedom for numerator and $n-v$ degrees of freedom for denominator, $F \sim F_{u, n-v},[1]$. 


\section{The energy transition path in Australia: interplay between Coal, Gas and Renewables}

In this section we study the interplay between coal and gas, coal and renewables and gas and renewables according to the UCRCD model or its possible reductions. The data come from the BP Statistical Review of World Energy 2017 [33], and refer to the annual consumption in MToe from 1965 to 2016 for coal, from 1969 to 2016 for gas, from 2001 to 2016 for wind, and from 2009 to 2016 for solar. It is important to observe that in the case of renewables, the data refer to onshore energy, since offshore is not substantial in Australia. Figure 1 shows the time series of coal, gas and renewables (wind and solar put together), where it is possible to see that coal has recently experienced a growth after some years of decline, gas is slowing down while renewables have been characterized by a steady growth. Notice that the year 1965 is not the starting point of coal consumption, but just the first available observation in our data set. One may easily appreciate large differences in scales referring to coal and gas on the one hand and renewables on the other, so that from a simple graphical inspection it is difficult to evaluate the presence of competition and more specifically the role played by renewables. Still, it can be noticed a huge break in coal consumption after 2000 and before 2010, likely related to the series of gas and renewables. Indeed, renewables entered the market around 2000 and feed-in tariffs were implemented since 2008, so the decline in coal observed before 2010 may be related to this. In order to statistically test this intuition and provide a quantitative measure of it, we fitted both a UCRCD model with $\delta=\gamma$ and with $\delta \neq \gamma$. On the basis of the tests for nested models illustrated in subsection 3.2, it has been found 
that the model with $\delta \neq \gamma$ significantly outperforms the reduced one with $\delta=\gamma$ just in the 'gas and renewables' case, since $\tilde{R}^{2}=0.16$ and $F=10.66$. Thus, the standard UCRCD with $\delta=\gamma$ has been employed in the "coal and gas' and 'coal and renewables' cases, and the UCRCD with $\delta \neq \gamma$ in the 'gas and renewables' case.

\subsection{Coal and gas}

The first application of the model refers to coal and gas consumption series, and the results are summarized in Table 2, Table 3 and Figure 2. The model reaches a satisfactory level of fitting, $R^{2}=0.98259$, even though parameter estimates $\hat{m}_{a}, \hat{p}_{1 a}$ and $\hat{q}_{1 a}$ referring to the stand-alone phase of coal are very unstable, as testified by the $95 \%$ confidence intervals. This is due to the fact that the series of coal is truncated at the beginning, which typically implies an instability of estimates. The remaining parameters are very stable and confirm the existence of a competitive dynamics between coal and gas. In particular, studying the within and cross imitation coefficients, summarized in Table 3, it may be noticed that both coal and gas have been characterized by a positive within imitation coefficient: for coal it is $\hat{q}_{1 c}+\hat{\delta}=0.078$, while for gas it is $\hat{q}_{2}=0.146$. Interestingly, the cross imitation coefficient is negative, although not strong, for both: the competitive effect exerted by gas on coal is $\hat{q}_{1 c}=-0.097$, while the effect of coal on gas is $\hat{q}_{2}-\hat{\delta}=-0.029$. The interplay between coal and gas is characterized by a proper competition, as corroborated by the negative value of the cross imitation coefficients, see Table 4. 


\subsection{Coal and renewables}

This subsection analyzes the role played by renewables with respect to coal in order to understand whether wind and solar are exerting a competitive effect on it and whether coal is hindering their diffusion. At a first step the standard UCRCD model was applied but parameters $p_{1 c}$ and $p_{2}$ were found not significant. A reduced model without parameters $p_{1 c}$ and $p_{2}$ was estimated, the results of which are summarized in Table 5, Table 6, Figure 3 and Figure 4. In particular, Figure 4 well shows that the decline in coal observed around 2000 and before 2010, seems to be evidently related to the uptake of renewables, as statistically proven through the model. It may be observed that the overall fitting is very good, $R^{2}=0.996234$, and parameter estimates are very stable except for $\hat{m}_{a}$ and $\hat{p}_{1 a}$ referred to the stand alone part of coal. In particular, the estimate of $m_{a}$ should not be trusted and its high value is due to fact that the series is truncated at the beginning. Interestingly, the non significance of parameters $p_{1 c}$ and $p_{2}$ indicates the absence of innovators. However, in the case of coal the innovative component has been already captured by parameter $p_{1 a}$, while for renewables the absence of innovative adopters is the sign that incentive measures and subsidies were necessary and effective in stimulating the take off of these technologies. The analysis of within and cross imitation coefficients, displayed in Table 6, yields very interesting results and indicates that there is a strong asymmetry in terms of effects. Renewables are experiencing a very strong growth as testified by the high value of the within imitation coefficient, $\hat{q}_{2}=0.604$, while the corresponding estimate for coal is quite weak, $\hat{q}_{1 c}+\hat{\delta}=0.071$, which is coherent with the recent declining trend of the series. The cross imitation 
parameters show that wind and solar are exerting a strongly negative effect on coal, $\hat{q}_{1 c}=-0.532$, while the reverse does not occur and coal is apparently not affecting the diffusion of renewables since $\hat{q}_{2}-\hat{\delta}=0.00003$.

The interplay between coal and renewables is characterized by a strong competitive effect of renewables on coal, as supported by the highly negative cross imitation coefficient, while the effect of coal on renewables is essentially zero, see Table 7 .

\subsection{Gas and renewables}

The last case involves the interplay between gas and renewables. Similarly to the case of 'coal and renewables', the UCRCD model (with $\delta \neq \gamma$ ) needed to be reduced by eliminating parameters $p_{1 c}$ and $p_{2}$, due to their non significance. The reduced model obtains a very high level of fitting, $R^{2}=0.994507$, and all parameter estimates are very stable. Results are outlined in Table 8, Table 9, Figure 5 and Figure 6. Parameters $m_{a}, p_{1 a}$ and $q_{1 a}$ referring to the stand alone phase of gas are well estimated. Interestingly, there has been a notable increase of the market potential from the stand alone phase $\left(\hat{m}_{a}=614\right)$ to the competition one $\left(\hat{m}_{c}=1331\right)$. This may be taken as a sign that the entrance of renewables has implied an overall enlargement of the market. It is worth observing that similar considerations cannot be done with coal because, as already explained, in this case the series is truncated at the beginning causing high instability of the stand alone market potential, $\hat{m}_{a}$. The absence of innovation parameters $p_{1 c}$ and $p_{2}$ may be interpreted similarly to the case of coal and renewables: parameter $p_{1 a}$ captures the innovative behavior within the gas series, while the absence of an innovative behavior for renewables reflects the need of incentive measures to stimulate 
their growth. The within and cross imitation coefficients suggest a very interesting situation, as outlined in Table 9. Renewables are characterized by a strong within imitation, $\hat{q}_{2}=0.463$, and by a positive, although very slight, cross imitation exerted by gas, $\hat{q}_{2}-\hat{\gamma}=0.0001$. This indicates that the diffusion of renewables has been driven by internal forces, but also by a positive, though limited, effect of gas. On the other hand, it may be observed that gas has been characterized by a limited within imitation, $\hat{q}_{1 c}+\hat{\delta}=0.070$ and a highly positive cross imitation coefficient $\hat{q}_{1 c}=1.648$. This result suggests that the growth of renewables is aiding the growth of gas, whose role as a transition fuel is therefore strongly confirmed. In Figure 5 the model fitting may be appreciated. The interplay between gas and renewables is characterized by a strong collaborative effect of renewables on gas, as testified by the highly positive cross imitation coefficient, while gas exerts a weaker but positive effect on renewables, see Table 10.

\section{Summary of results and conclusions}

The expression "energy transition" indicates a long-term structural change in energy systems. It typically refers to a regime shift from fossil fuel based systems to renewable energy technologies, and a shift from centralized to decentralized production of energy, [1]. In recent years, there has been a growing interest towards the energy transition in Australia, as an increasing body of literature testifies. Indeed, Australia's specificities are making the transition a very challenging and worth of investigation process: the great endowment of both fossil fuels and natural resources, a historically strong reliance on carbon generated electricity, a contradictory behaviour of politics 
that has sustained the fossil fuel industry while implementing incentive programs for renewables, a market dominance of coal and gas challenged by a rapid increase of renewables are all elements of an extremely attracting case study. To our knowledge, the literature so far developed on the topic has mainly concentrated on qualitative and policy-oriented analyses. To mention some recent contributions, Cheung and Davies in [14] have highlighted the historical contradictions of the Australian energy policy, Poruschi et al. in [5] have reviewed the existing feed-in tariffs mechanisms, underlying their fragmentation. Hudson in [34] has explained the apparent hostility of policy makers towards solar and wind with ideological factors, while Simpson in [35] performed a socio-technical analysis in order to understand the role of network operators in facilitating or hindering the implementation of a distributed generation network.

The study here presented, with its data-oriented approach based on innovation diffusion models, aims to bring a quantitative and complementary perspective within the existing literature on transition to sustainable energy in Australia. Our analysis has been focused on providing a market interpretation of the observed data patterns, so that it has more a descriptive power rather than a predictive one. Still, the model employed is able to capture market mechanisms and interactions that in no way would have been evident by a simple graphical inspection of data. Going back to Figure 1, one may clearly see that coal and gas dominate the market and a substitution effect between coal and gas may be noticed in the last 3-4 years, which seems to suggest that gas is gradually replacing coal. At first sight, the contribution of renewables appears quite limited, because in absolute terms their market 
share is small with respect to fossil fuels. So, trying to infer the competitive strength of renewables from the raw analysis of data could lead to misleading conclusions. Starting from this consideration, a model-based analysis of the evolutionary dynamics of coal, gas and renewables has appeared a more compelling choice, shedding light on the existence, the intensity and nature (competition or collaboration) fo their interplay. In fact, one of the most powerful aspects of the UCRCD model is the possibility to have a cross imitation coefficient, describing the interaction effect, that may be either negative or positive. Typically, cross imitation has a negative sign and indicates a competitive relationship; however, in some interesting cases, it also presents a positive sign, that is a collaborative relationship.

Overall, the most important result deriving from the analysis, is the statistical significance of the UCRCD model in all the three cases considered: this empirically proves the existence of a significant interplay between coal, gas and renewables, and specifically confirms that renewables play a influential role on fossil fuels, despite their quite limited market share.

Moreover, the interpretation of the imitation parameters offers important insights. In all cases, the within imitation coefficient is positive, although strongly positive just in the renewable case. Instead, it has a much more limited entity in coal and gas, which is coherent with the declining or stalling trend of these sources. Incidentally, one may notice that the model well captures the declining path of coal: even if this is not a particularly exciting finding per se, it is interesting to observe that the rise in coal consumption occurred in years 2014-15, likely deriving from the revocation of the carbon pricing mechanism, did not have any effect on the general trend of the series. 
This seem to suggest that some defensive policy choices implemented to keep a "business as usual" environment had more an ideological than practical meaning. On the contrary, the high within imitation coefficient characterizing the diffusion of renewables diagnoses the efficacy of the RET and of feed-in tariff mechanisms. This is also confirmed by the absence of innovation parameters for renewables, requiring a reduction of the UCRCD model: the role of innovators has been played by incentive measures, without which the uptake process would have been significantly slower.

However, the most interesting conclusions are drawn by studying the cross imitation coefficients. As already mentioned, the negative cross imitation coefficient in the coal-gas application proves a real competition between the two, and confirms that gas is playing the role of a direct substitute of coal. Regarding the applications with renewables, the findings show that the cross imitation coefficient towards coal is highly negative, proving that renewables are really able to compete with coal. On the other hand, we notice that the relationship between coal and renewables is highly asymmetric, because coal does not exert any effect on the diffusion of renewables, as testified by the almost negligible value of its cross imitation coefficient. The model suggests that the diffusion of renewables implies a regime change with an "independent" path.

Probably the most intriguing result is that concerning the gas-renewables relationship: in this case the cross imitation coefficients are found to be positive, proving a collaborative relationship. Specifically, the diffusion of renewables exerts a clear and extremely positive effect on gas, aiding its key role as a transition mean. Instead, the position of gas towards renewables seems 
to be slightly ambivalent: the positive but low value of its cross imitation coefficient suggests that gas collaborates with renewables towards transition and coal replacement, but it also represents a mean to keep the status quo and continue business as usual. So, the empirical evidence confirms the expectation that gas will play a major role in regime change, as recommended by the Finkel Review, but also calls the attention on the risk that more gas could simply mean less renewables.

\subsection{Conclusions}

According to the literature on socio-technical transitions recalled in Simpson [35], a regime change can occur following a "niche-based model" or a "transition management model". In the first case the technology is able to disrupt the incumbent regime and drive the change, in the second there is a "guiding vision" that influences the process. The analysis developed by Simpson suggests that the second model would be the most effective to realize a transition towards a decentralized electricity system. Our analysis seems coherent with this view, because we have empirically proven that the competitive strength of renewables derives from a fast diffusion process due to focused policies. At the same time, we also acknowledge the power of continuing descrease in renewable energy generation costs that have driven investment in renewables in recent years.

Less fragmented policies and a unified vision of Australian future energy appear a basic need for a successful transition. The need for a unified vision is further motivated by externalities such as the increasing global consensus on carbon abatement, which Australia cannot ignore. The model-based ap- 


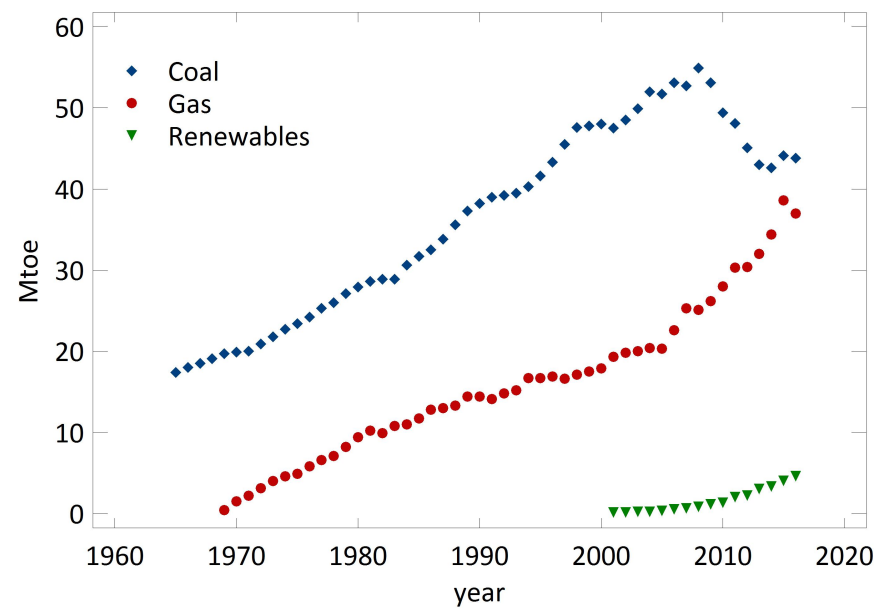

Figure 1: Annual consumption of coal, gas and renewables (wind and solar together) in MToe. Data source: BP Statistical Review of World Energy 2016.

proach to energy transition in this paper provides a good starting point for descriptive and impartial analysis of energy transition trends even under pol555 icy uncertainties and structural issues and represent a benchmark for other 556 studies focusing on Australia. 
Table 1: Sign of cross imitation coefficient and their competition-collaboration interpretation

\begin{tabular}{c|c|l}
\hline$q_{1} c$ & $q_{2}-\gamma$ & Interpretation \\
\hline negative & negative & full competition \\
negative & positive & 2 competes with 1,1 collaborates with 1 \\
positive & negative & 2 collaborates with 1,1 competes with 2 \\
positive & positive & full collaboration \\
\hline
\end{tabular}

Table 2: Coal and gas: parameter estimates of standard UCRCD model; $95 \%$ CIs into brackets. Estimates performed on instantaneous data.

\begin{tabular}{c|c|c|c|c}
\hline$m_{a}$ & $p_{1 a}$ & $q_{1 a}$ & $m_{c}$ & $q_{1 c}$ \\
\hline 1814 & 0.009 & 0.041 & 5828 & -0.097 \\
$(-359705)$ & $(-1.835)$ & $(-2.335)$ & $(5215)$ & $(-0.120)$ \\
$(363335)$ & $(1.854)$ & $(2.418)$ & $(6442)$ & $(-0.074)$ \\
\hline$q_{2}$ & $\delta$ & $p_{1 c}$ & $p_{2}$ & $R^{2}$ \\
\hline 0.146 & 0.175 & 0.0015 & 0.0015 & 0.98259 \\
$(0.123)$ & $(0.143)$ & $(0.0012)$ & $(0.0011)$ & \\
$(0.169)$ & $(0.207)$ & $(0.0019)$ & $(0.0018)$ & \\
\hline
\end{tabular}


Table 3: Interplay between coal and gas: within and cross imitation coefficients.

\begin{tabular}{c|cc}
\hline & Coal & Gas \\
\hline Within & 0.078 & 0.146 \\
& $\hat{q}_{1 c}+\hat{\delta}$ & $\hat{q}_{2}$ \\
\hline Cross & -0.097 & -0.029 \\
& $\hat{q}_{1 c}$ & $\hat{q}_{2}-\hat{\delta}$ \\
\hline
\end{tabular}

Table 4: Coal and gas: sign of cross imitation coefficients and their competitioncollaboration interpretation.

\begin{tabular}{c|c|l}
\hline$q_{1} c$ & $q_{2}-\delta$ & Interpretation \\
\hline negative & negative & full competition \\
\hline
\end{tabular}

Table 5: Coal and renewables: parameter estimates of reduced UCRCD model with $p_{1 c}=0$ and $p_{2}=0 ; 95 \%$ CIs into brackets. Estimates performed on instantaneous data.

\begin{tabular}{c|c|c|c}
\hline$m_{a}$ & $p_{1 a}$ & $q_{1 a}$ & $m_{c}$ \\
\hline 11902 & 0.0013 & 0.033 & 2890 \\
$(-3577)$ & $(-0.0003)$ & $(0.027)$ & $(2771)$ \\
$(27382)$ & $(0.0031)$ & $(0.039)$ & $(3009)$ \\
\hline$q_{1 c}$ & $q_{2}$ & $\delta$ & $R^{2}$ \\
\hline-0.532 & 0.604 & 0.604 & 0.996234 \\
$(-0.719)$ & $(0.419)$ & $(0.419)$ & \\
$(-0.346)$ & $(0.789)$ & $(0.790)$ & \\
\hline
\end{tabular}




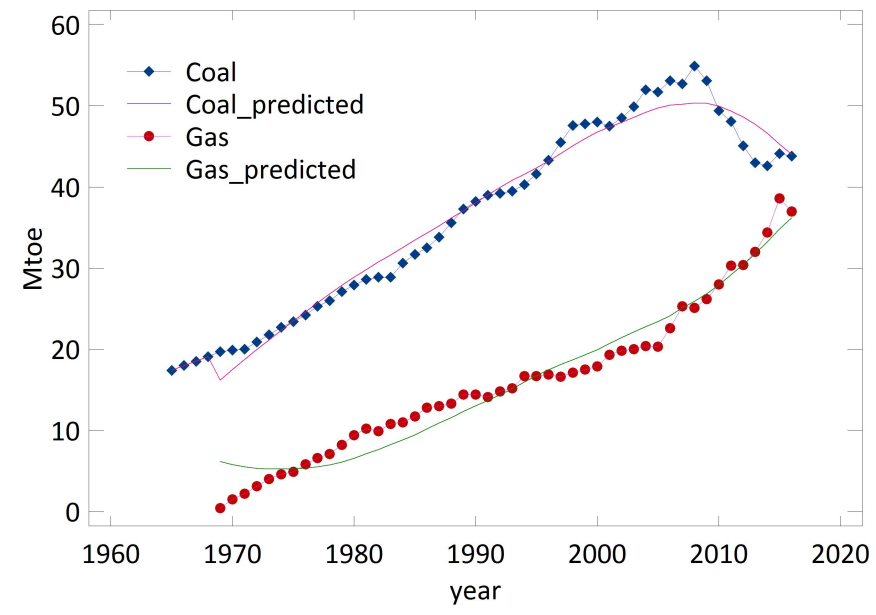

Figure 2: Annual consumption of coal and gas and fitted UCRCD model.

Table 6: Interplay between coal and renewables: within and cross imitation coefficients.

\begin{tabular}{c|cc}
\hline & Coal & Renewables \\
\hline Within & 0.071 & 0.604 \\
& $\hat{q}_{1}+\hat{\delta}$ & $\hat{q}_{2}$ \\
\hline Cross & -0.532 & 0.00003 \\
& $\hat{q}_{1 c}$ & $\hat{q}_{2}-\hat{\delta}$ \\
\hline
\end{tabular}


Table 7: Coal and renewables: sign of cross imitation coefficients and their competitioncollaboration interpretation.

\begin{tabular}{c|c|l}
\hline$q_{1 c}$ & $q_{2}-\delta$ & Interpretation \\
\hline negative & positive & $\begin{array}{l}\text { renewables compete with coal, } \\
\text { coal does not affect renewables }\end{array}$ \\
\hline
\end{tabular}

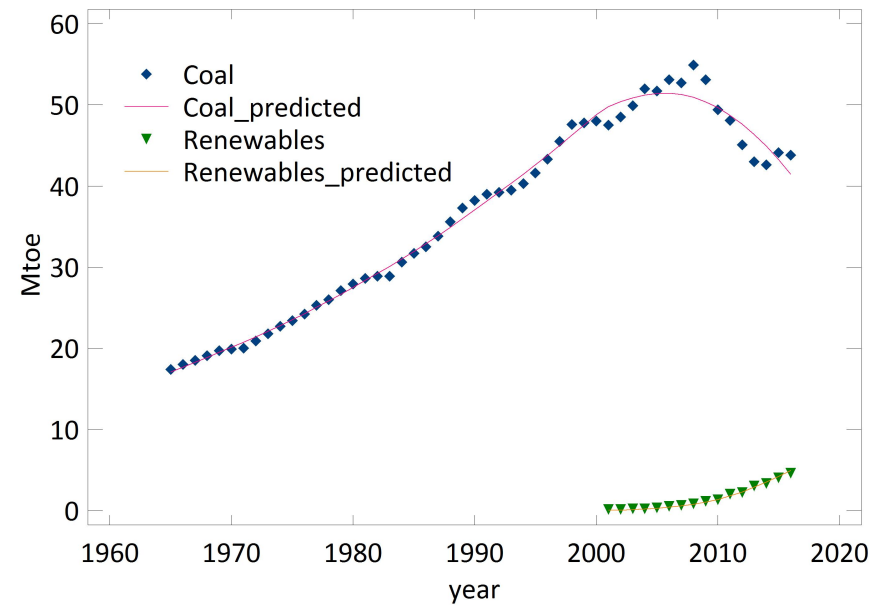

Figure 3: Annual consumption of coal and renewables and fitted reduced UCRCD model with $p_{1 c}=0$ and $p_{2}=0$. 


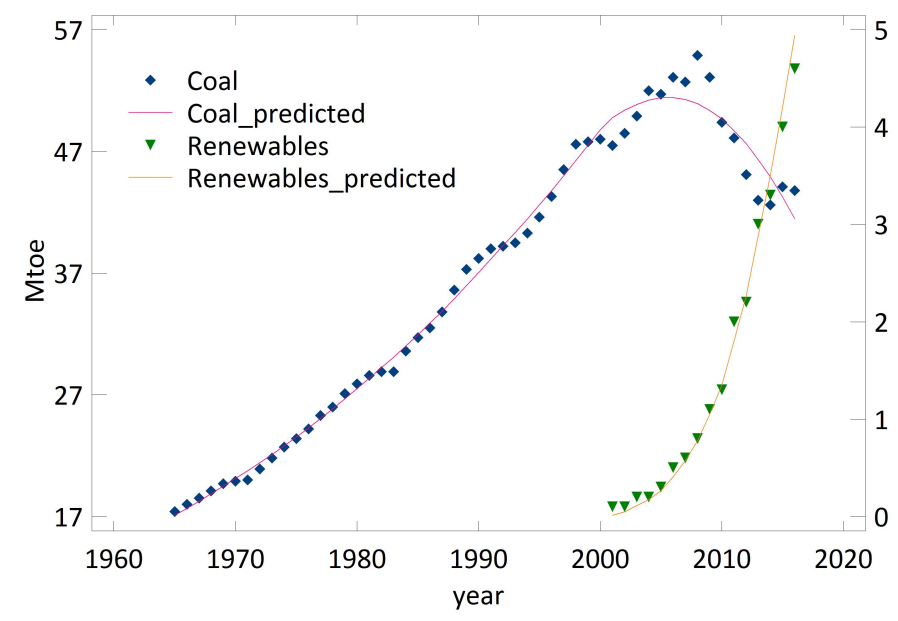

Figure 4: Rescaled annual consumption of coal and renewables and fitted reduced UCRCD model with $p_{1 c}=0$ and $p_{2}=0$. 
Table 8: Gas and renewables: parameter estimates of reduced UCRCD model with $p_{1 c}=0$ and $p_{2}=0 ; 95 \%$ CIs into brackets. Estimates performed on instantaneous data.

\begin{tabular}{c|c|c|c}
\hline$m_{a}$ & $p_{1 a}$ & $q_{1 a}$ & $m_{c}$ \\
\hline 614 & 0.004 & 0.102 & 1331 \\
$(555)$ & $(0.003)$ & $(0.092)$ & $(947)$ \\
$(672)$ & $(0.005)$ & $(0.111)$ & $(1715)$ \\
\hline$q_{1 c}$ & $q_{2}$ & $\delta$ & $\gamma$ \\
\hline 1.648 & 0.463 & -1.577 & 0.463 \\
$(0.323)$ & $(0.234)$ & $(-2.895)$ & $(0.232)$ \\
$(2.972)$ & $(0.693)$ & $(-0.260)$ & $(0.694)$ \\
\hline
\end{tabular}

Table 9: Interplay between gas and renewables: within and cross imitation coefficients.

\begin{tabular}{c|cc}
\hline & Gas & Renewables \\
\hline Within & 0.070 & 0.463 \\
& $\hat{q}_{1}+\hat{\delta}$ & $\hat{q}_{2}$ \\
\hline Cross & 1.648 & 0.00011 \\
& $\hat{q}_{1}$ & $\hat{q}_{2}-\hat{\gamma}$ \\
\hline
\end{tabular}


Table 10: Coal and renewables: sign of cross imitation coefficients and their competitioncollaboration interpretation.

\begin{tabular}{c|c|l}
\hline$q_{1} c$ & $q_{2}-\gamma$ & Interpretation \\
\hline positive & positive & $\begin{array}{l}\text { renewables collaborate with gas, } \\
\text { gas (slightly) collaborates with renewables }\end{array}$ \\
\hline
\end{tabular}

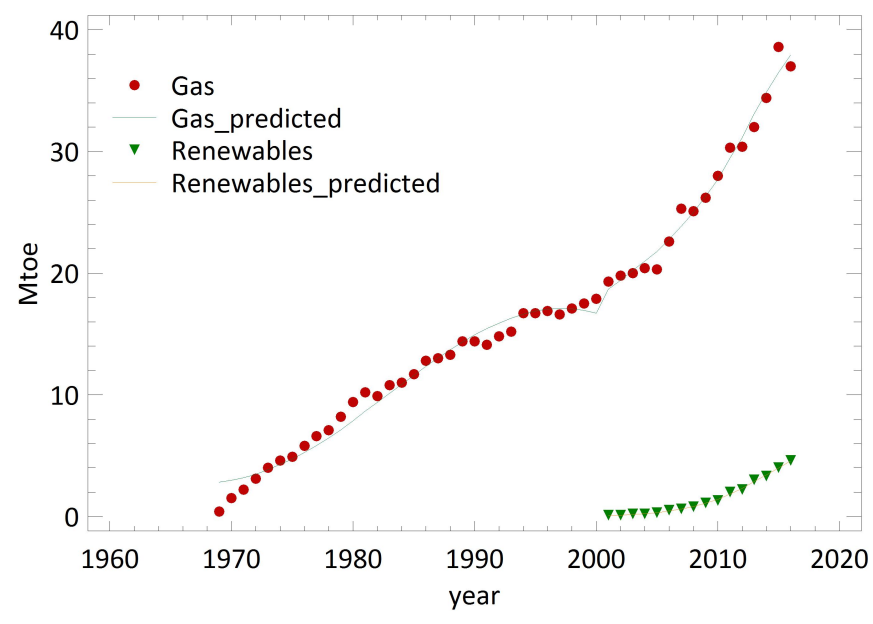

Figure 5: Annual consumption of gas and renewables and fitted reduced UCRCD model with $p_{1 c}=0$ and $p_{2}=0$. 


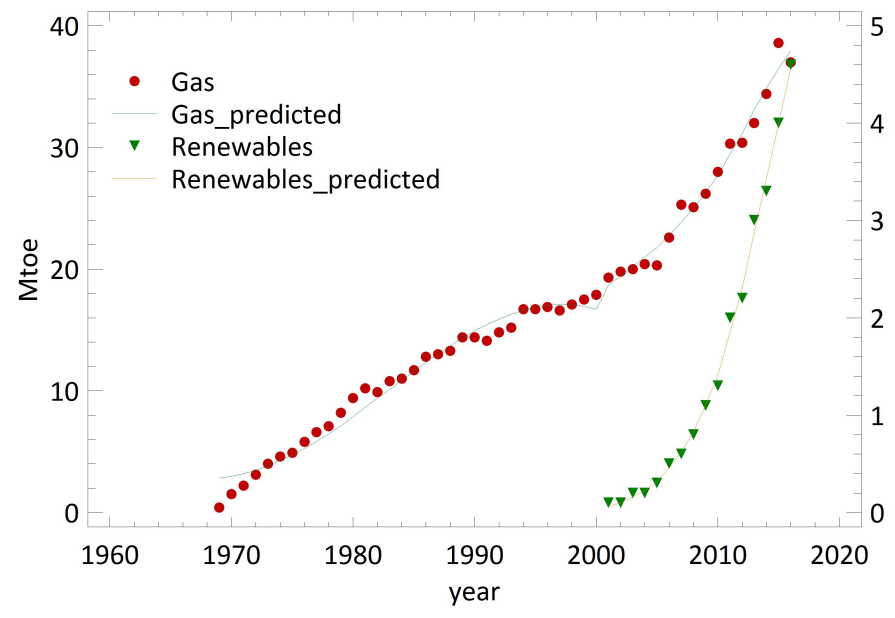

Figure 6: Rescaled annual consumption of gas and renewables and fitted reduced UCRCD model with $p_{1 c}=0$ and $p_{2}=0$. 


\section{References}

[1] M Guidolin and R Guseo. The German energy transition: Modeling competition and substitution between nuclear power and renewable energy technologies. Renewable and Sustainable Energy Reviews, 60:1498$1504,2016$.

[2] KS Stegen and M Seel. The winds of change: How wind firms assess Germany's energy transition. Energy Policy, 61:1481-1489, 2013.

[3] L Byrnes, C Brown, J Foster, and LD Wagner. Australian renewable energy policy: Barriers and challenges. Renewable Energy, 60:711-721, 2013.

[4] Y Hua, M Oliphant, and EJ Hu. Development of renewable energy in Australia and China: A comparison of policies and status. Renewable Energy, 85:1044-1051, 2016.

[5] L Poruschi, CL Ambrey, and JCR Smart. Revisiting feed-in tariffs in Australia: A review. Renewable and Sustainable Energy Reviews, 82:260-270, 2018.

[6] Australian Energy Statistics. Australian Energy Update 2018, 2018.

[7] International Energy Agency. Energy Policies of IEA Countries: Australia 2018. 2018.

[8] L Molyneaux, C Froome, L Wagner, and J Foster. Australian power: Can renewable technologies change the dominant industry view? Renewable Energy, 60:215-221, 2013. 
[9] P Effendi and J Courvisanos. Political aspects of innovation: Examining renewable energy in Australia. Renewable Energy, 38(1):245-252, 2012.

[10] MJ Brear, M Jeppesen, D Chattopadhyay, C Manzie, T Alpcan, and R Dargaville. Least cost, utility scale abatement from Australia's NEM (national electricity market). part 2: Scenarios and policy implications. Energy, 101:621-628, 2016.

[11] M Jeppesen, MJ Brear, D Chattopadhyay, C Manzie, R Dargaville, and T Alpcan. Least cost, utility scale abatement from Australia's NEM (national electricity market). part 1: Problem formulation and modelling. Energy, 101:606-620, 2016.

[12] B Warren, P Christoff, and D Green. Australia's sustainable energy transition: the disjointed politics of decarbonisation. Environmental Innovation and Societal Transitions, 21:1-12, 2016.

[13] BK Sovacool. How long will it take? Conceptualizing the temporal dynamics of energy transitions. Energy Research \& Social Science, 13:202$215,2016$.

[14] G Cheung and PJ Davies. In the transformation of energy systems: what is holding Australia back? Energy Policy, 109:96-108, 2017.

[15] FW Geels, BK Sovacool, T Schwanen, and S Sorrell. The socio-technical dynamics of low-carbon transitions. Joule, 1(3):463-479, 2017.

[16] C Furlan and C Mortarino. Forecasting the impact of renewable energies in competition with non-renewable sources. Renewable and Sustainable Energy Reviews, 81:1879-1886, 2018. 
[17] AD Mitchell and J Casben. Natural Resources and Energy Regulation in Australia: The Energy White Paper in Context. In Emerging Issues in Sustainable Development, pages 3-25. Springer, 2016.

[18] A Finkel. Independent Review into the Future Security of the National Electricity Market, 2017.

[19] C Marchetti. Primary energy substitution models: on the interaction between energy and society. Technological Forecasting and Social Change, 10(4):345-356, 1977.

[20] C Marchetti. Society as a learning system: discovery, invention, and innovation cycles revisited. Technological Forecasting and Social change, 18(4):267-282, 1980 .

[21] C Marchetti and N Nakicenovic. The dynamics of energy systems and the logistic substitution model. 1979.

[22] KU Rao and VVN Kishore. A review of technology diffusion models with special reference to renewable energy technologies. Renewable and Sustainable Energy Reviews, 14(3):1070-1078, 2010.

[23] N Meade and T Islam. Modelling European usage of renewable energy technologies for electricity generation. Technological Forecasting and Social Change, 90:497-509, 2015.

[24] SW Davies and I Diaz-Rainey. The patterns of induced diffusion: Evidence from the international diffusion of wind energy. Technological Forecasting and Social Change, 78(7):1227-1241, 2011. 
[25] FM Bass. A new product growth for model consumer durables. Management science, 15(5):215-227, 1969.

[26] FM Bass, TV Krishnan, and DC Jain. Why the Bass model fits without decision variables. Marketing science, 13(3):203-223, 1994.

[27] R Guseo and C Mortarino. Correction to the paper "Optimal product launch times in a duopoly: balancing life-cycle revenues with product cost". Operations research, 58(5):1522-1523, 2010.

[28] R Guseo and C Mortarino. Sequential market entries and competition modelling in multi-innovation diffusions. European Journal of Operational Research, 216(3):658-667, 2012.

[29] R Guseo and C Mortarino. Within-brand and cross-brand word-ofmouth for sequential multi-innovation diffusions. IMA Journal of Management Mathematics, 25(3):287-311, 2014.

[30] TV Krishnan, FM Bass, and V Kumar. Impact of a late entrant on the diffusion of a new product/service. Journal of marketing research, $37(2): 269-278,2000$.

[31] S Savin and C Terwiesch. Optimal product launch times in a duopoly: Balancing life-cycle revenues with product cost. Operations Research, $53(1): 26-47,2005$.

[32] GAF Seber and CJ Wild. Nonlinear regression: Wiley series in probability and mathematical statistics, 1989. 
645

646

647

648

649

650

651

652

[33] Historical Data Workbook. BP Statistical Review of World Energy. 2017.

[34] M Hudson. Wind beneath their contempt: Why Australian policymakers oppose solar and wind energy. Energy Research 83 Social Science, 28:11-16, 2017.

[35] G Simpson. Network operators and the transition to decentralised electricity: An Australian socio-technical case study. Energy Policy, 110:422-433, 2017. 